\title{
The gradual loss of vision
}

\section{N Du Toit MBChB, DipOphth(SA), FRCS(Ed), FCOphth(SA), MMed}

To cite this article: N Du Toit MBChB, DipOphth(SA), FRCS(Ed), FCOphth(SA), MMed (2013) The gradual loss of vision, South African Family Practice, 55:6, 493-500, DOI: 10.1080/20786204.2013.10874404

To link to this article: http://dx.doi.org/10.1080/20786204.2013.10874404
ใ (c) 2013 SAAFP. Published by Medpharm.

\section{Published online: 15 Aug 2014.}

Submit your article to this journal $\sqsubset$

\section{山 Article views: 841}

Q View related articles $\square$ 


\title{
The gradual loss of vision
}

Du Toit N, MBChB, DipOphth(SA), FRCS(Ed), FCOphth(SA), MMed Consultant Ophthalmic Surgeon, Division of Ophthalmology, Groote Schuur Hospital; Senior Lecturer, University of Cape Town Correspondence to: Nagib du Toit, e-mail: nagib.dutoit@uct.ac.za Keywords: gradual, loss of vision, cataracts, refractive error, optic atrophy, glaucoma, age-related macular degeneration

\begin{abstract}
Gradual loss of vision is a clinical problem that is encountered fairly regularly in most primary healthcare settings. Patients present with chronic, slowly progressive and generally painless visual loss. The reduction in vision is usually bilateral, though frequently asymmetrical, and occurs over weeks to years. A goal-directed assessment of the patient presenting with gradual loss of vision is required. A history of the type of visual loss, e.g. central or peripheral, and whether it is worse for near vision or distance vision, is helpful. The examination should focus on visual acuity, confrontation visual field testing, pupil testing for the presence of an afferent pupil defect and assessment of the red reflex and fundoscopy. The more common conditions that cause gradual loss of vision can be divided into two groups based on the reversibility of the visual loss. Cataracts, refractive error, corneal blindness and early diabetic macular oedema are generally reversible. Optic atrophy, glaucoma, retinal degeneration and age-related macular degeneration usually cause permanent loss of vision. Most of these conditions are briefly discussed in this article. This has been performed at a level that is suitable to primary care.
\end{abstract}

(P) Peer reviewed. (Submitted: 2013-12-19 Accepted: 2013-04-10). @ SAAFP

S Afr Fam Pract 2013;55(6):493-500

\section{Introduction}

Patients with gradual loss of vision present with chronic, slowly progressive loss of vision which is generally painless. Visual loss is usually bilateral, but may occur asymmetrically, and happens over weeks to years. Poor vision in one eye may only be noticed when the patient closes the other eye, and thus may be reported to be sudden in onset in some cases. At this stage, it is important to note that blindness is defined as visual acuity worse that $3 / 60$ in both eyes, or a visual field restricted to less than 10 degrees around central fixation bilaterally. Visual impairment is divided into different categories, but generally refers to visual acuity worse than $6 / 18$ in the better eye. ${ }^{1}$ The need for an update in this field became apparent during a recent study on primary health eye care knowledge by general practitioners. ${ }^{2}$ This article is the third in a series which attempts to address this issue.

\section{Approach to the patient}

A goal-directed assessment of the patient who presents with gradual loss of vision is required. A history of the type of visual loss, e.g. central or peripheral, and whether it is worse nearby or at distance is helpful. Central loss is found with lesions of the macula, while peripheral loss is found in glaucoma. Patients with myopia and certain retinal degeneration may experience poor vision with dim light, and those with a cataract may find bright light to affect the vision more, although this symptom depends on the morphology of the lens opacity. The examination should focus on visual acuity, visual field testing, pupil testing for the presence of an afferent pupil defect, and assessment of the red reflex and fundoscopy. Many of the conditions discussed will initially cause reversible visual loss, but a few may become permanent if they are not appropriately managed. ${ }^{3}$

The more common conditions that cause gradual loss of vision can be divided into two groups, based on the reversibility of the visual loss. The conditions in these two categories are listed in Table I. These will be discussed later.

Table I: The common causes of gradual loss of vision

\begin{tabular}{l} 
Reversible causes \\
Cataract \\
Refractive error \\
Corneal blindness \\
Diabetic macular oedema \\
\hline Irreversible causes \\
\hline Optic atrophy \\
Glaucoma \\
Age-related macular degeneration \\
\hline Retinitis pigmentosa
\end{tabular}




\section{VA improves} markedly with Pinhole testing:

»Refractive error<smiles>[13CH3]</smiles>

\section{VA may be:}

-Worse for distance

»Myopia

-Worse for near

»Hypermetropia

(simplified)

$$
\begin{aligned}
& \text { APD = Afferent Pupillary Defect } \\
& \text { VA }=\text { Visual Acuity }
\end{aligned}
$$

\section{Gradual loss of vision}

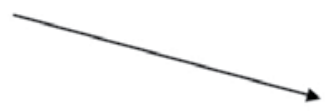

\section{(may be reversible)}

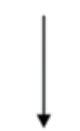

\section{Reduced red} reflex:

- Iris details not visible i.e. corneal opacity

»Corneal blindness

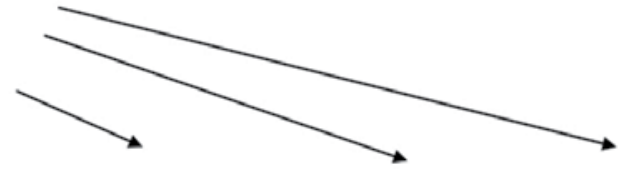

Reduced red reflex:

-Iris details are visible with opacification in pupil area i.e. lens opacity „Cataract (reduction of red reflex may vary from mild to complete whitening of the pupil $=$ leucocoria)

\section{Diabetic retinopathy with macula involved: \\ ") Diabetic maculopathy \\ (NB. May be reversible in early stages only)}

Macular
drusen or
pigmentary
changes:
"Age-related
Macular
degeneration
(NB. These
changes are
usually
irreversible,
but
improvement
is possible in
certain cases
with anti-
vascular
growth factor
treatment)

Macula drusen or pigmentary changes:

\section{"Age-related optic atrophy: Increased cup- to-disc ratio}

»Glaucoma

-Bone spicules in periphery

»Retinitis pigmentosa

-Associated disc swelling „Papilloedema

- Other causes of atrophy e.g. compression

APD: afferent papillary defect, VA: visual acuity

Figure 1: Flow chart for the primary care assessment of patients with gradual loss of vision

The more common conditions will be discussed in more detail than the less common ones.

It is important to note that most of these conditions cause bilateral visual loss, but they may be asymmetrical and initially affect one eye more than the other. Using the reversibility of the visual loss and the presence of an afferent pupil defect as the primary differentiating factors, with associated symptoms and signs as secondary, the causes can be narrowed down as indicated (Figure 1).

\section{Clinical conditions}

\section{Cataract}

A cataract is probably the most common cause of gradual visual loss. ${ }^{1}$ It refers to "clouding" or loss of clarity of the crystalline lens, with a resultant decrease in acuity. $A$ cataract may occur congenitally, but senile cataracts are much more common, and almost everyone over 70 years of age has some degree of lens opacity. ${ }^{3}$ The other causes of cataracts include diabetes, trauma, steroid therapy and chronic uveitis. Congenital cataracts may be familial, metabolic or idiopathic. Nuclear sclerotic, cortical and posterior subcapsular opacities are the most common morphological types in age-related cases.

A cataract is a very common cause of blindness, ${ }^{4-6}$ but patients may initially experience glare (with the cortical

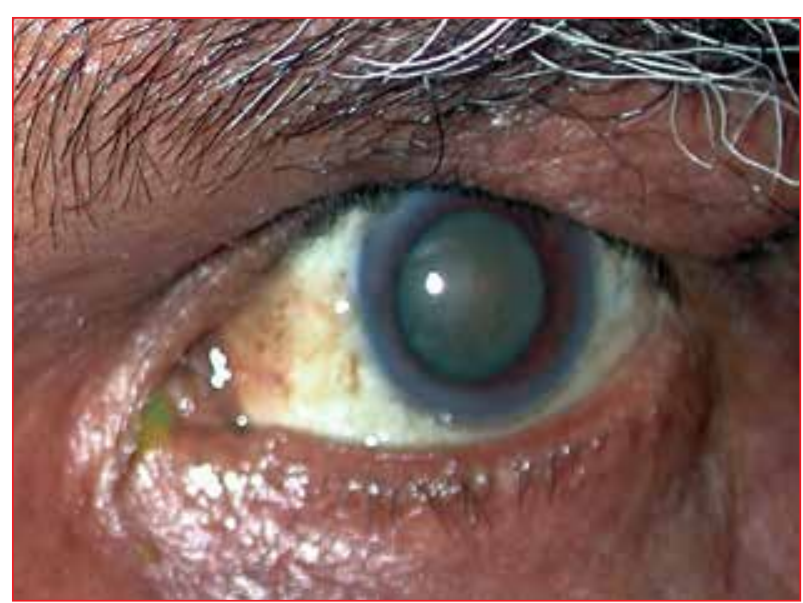

Source: The Division of Ophthalmology, University of Cape Town

Figure 2: Mature cataract (with absent red reflex) visible through the dilated pupil 
type), monocular diplopia or a change in refraction, usually a "myopic shift" because of nuclear sclerosis. Cataracts are recognised by a decrease in the red reflex (Figure 2), with resultant poor visualisation of the retina on attempted fundoscopy. It is important to exclude other co-existing ocular pathologies which may limit the chances of visual recovery after surgery. A cataract is also the most common cause of leukocoria (white pupil). ${ }^{3}$ Cataract blindness is reversible by microsurgery which aims to improve visual function and is safe, quick and cost-effective.

Cataract extraction can be performed in two basic ways, viz. intracapsular cataract extraction (ICCE), whereby the lens is removed together with its capsule, and extracapsular cataract extraction (ECCE), whereby the lens is removed leaving the capsule behind, which is the preferred technique.

This can be further subdivided into:

- Traditional ECCE: A large incision (usually limbal) (Figure 3) is made. A needle is used to make a hole in the lens capsule, and the cataract is expressed by putting pressure on the globe.

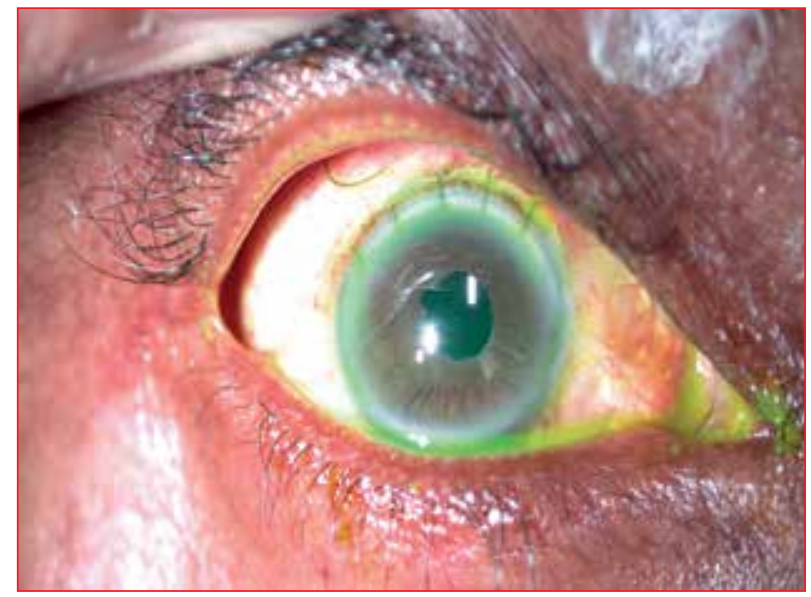

Source: The Division of Ophthalmology, University of Cape Town

Figure 3: Large limbal wound of traditional extracapsular cataract extraction

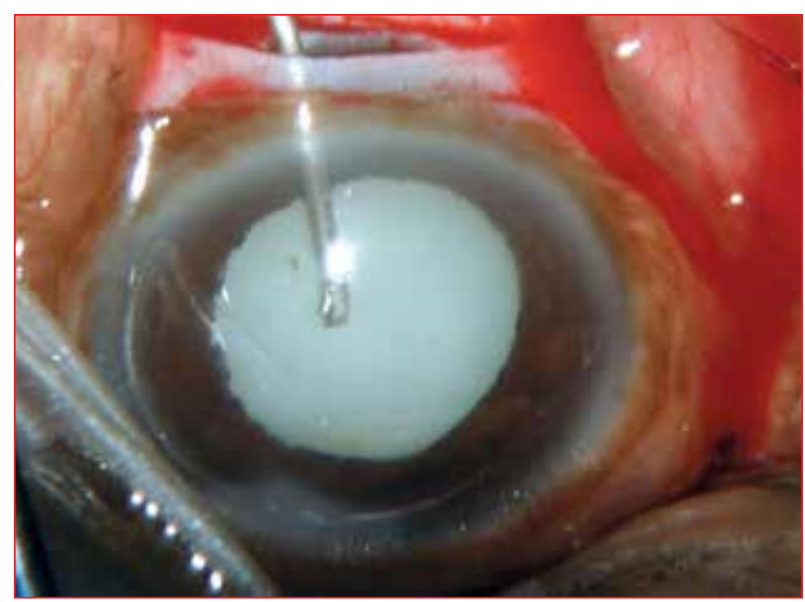

Source: The Division of Ophthalmology, University of Cape Town

Figure 4: Smaller scleral wound of small-incision extracapsular cataract extraction
- Small-incision ECCE: A smaller (usually scleral) (Figure 4) incision is used.

- Phacoemulsification: An ultrasound probe (Figure 5) is used to emulsify the cataract and aspirate it through a small scleral or corneal wound which needs no sutures. This surgical technique requires a shorter recovery time. ${ }^{3}$ More recently, femtosecond laser has been introduced to perform some of the steps of the procedure.

Patients should be referred for surgery when the visual disturbance interferes appreciably with the patient's lifestyle. ${ }^{1}$

\section{Refractive error}

This is also a common cause of blindness, ${ }^{4,6}$ and is correctable with spectacles. Images, caused by refraction at the cornea and lens, are formed either in front of, or behind, the retina. Light (parallel rays) from a distant object is focused on the retina in emmetropia (normal sight). Accommodation occurs when diverging rays from a near object are focused on the retina by thickening of the lens, which occurs when the ciliary muscle contracts and the lens zonules relax, causing the lens to become more spherical in shape.

With hypermetropia (long-sightedness), parallel rays from distant objects are focused behind the retina. This is corrected with convex lenses. Myopia (short-sightedness) causes parallel rays to be focused in front of the retina, and is corrected with concave lenses. Degenerative myopia occurs when patients with high degrees of myopia have "stretchy eyeballs", which may become progressively longer with time. They develop "cracks" in the choroid, and lose vision because of haemorrhage from neovascularisation around these cracks. ${ }^{3}$ Retinal degeneration may also be a cause of loss of vision in high myopes, in addition to the earlier onset of cataracts and glaucoma. ${ }^{7}$

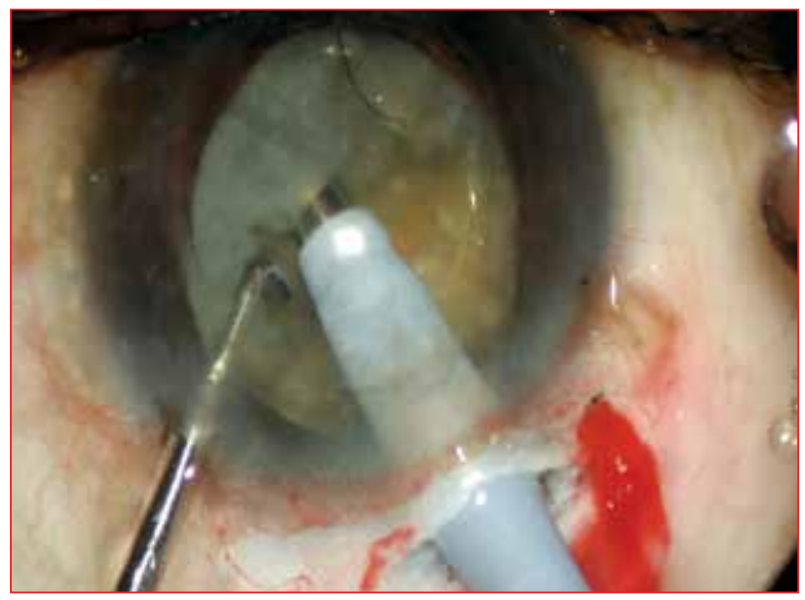

Source: The Division of Ophthalmology, University of Cape Town

Figure 5: Ultrasound probe used for phacoemulsification 


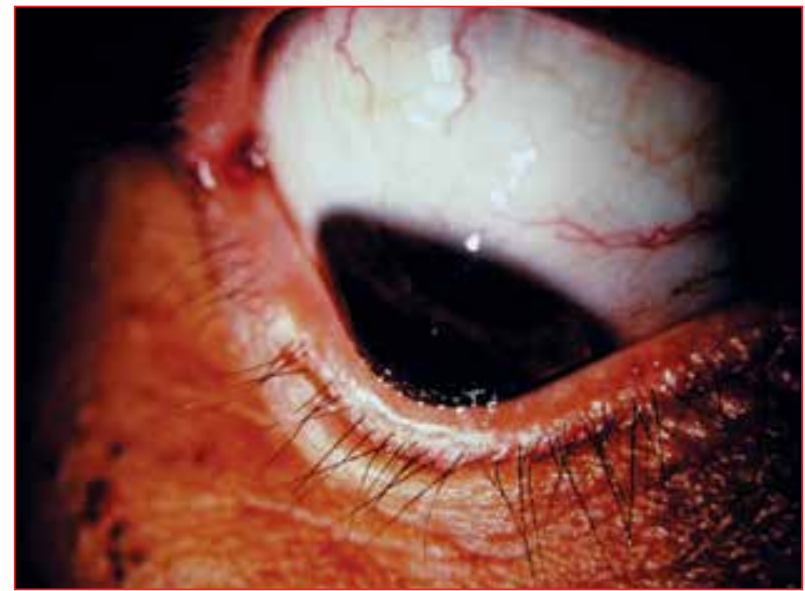

Source: The Division of Ophthalmology, University of Cape Town

Figure 6: Conically shaped cornea apparent in a downward gaze

Presbyopia occurs with ageing in the mid forties, because of loss of the ability of the lens to accommodate. Patients need convex lens correction for reading or near work. Astigmatism usually results from different curvatures in the two refracting planes of the cornea, causing an image from each plane to be formed at two different points in front of the retina, behind the retina, or both in front of and behind the retina. Keratoconus, which leads to progressive myopic astigmatism, is defined as a conically-shaped cornea (Figure 6). It has numerous associations. Atopy, eye rubbing and Down's syndrome are common associations. ${ }^{3}$ Keratoconus is treated initially with hard contact lenses, and later corneal cross-linking or corneal transplantation.

Any patient complaining of poor vision should have visual acuity measured with a 1-mm pinhole. Only parallel rays of light pass through the pinhole centrally, so that the eye does not have to refract them to obtain a clear retinal image. If the patient has a refractive error, vision will improve with the pinhole and the patient should be referred to an optometrist for spectacles. If the patient already has glasses, the pinhole may be used over them, and if there is further improvement, the patient will need to change his or her spectacles. ${ }^{1}$ Contact lenses are useful for greater refractive errors, but meticulous lens care is needed to avoid complications. Excimer laser correction of refractive errors should be reserved for those who are intolerant of glasses and contact lenses. ${ }^{3}$

\section{Corneal blindness}

Conditions which lead to opacification of the cornea will cause a reduction in visual acuity. These include trachoma in equatorial countries, which leads to entropion, trichiasis and scarring of the cornea; interstitial keratitis following infections, such as congenital syphilis, tuberculosis and measles; and climatic droplet keratopathy (Figure 7), which is caused by prolonged exposure to the elements, and is common in the elderly from the Eastern Cape. ${ }^{3}$ Corneal

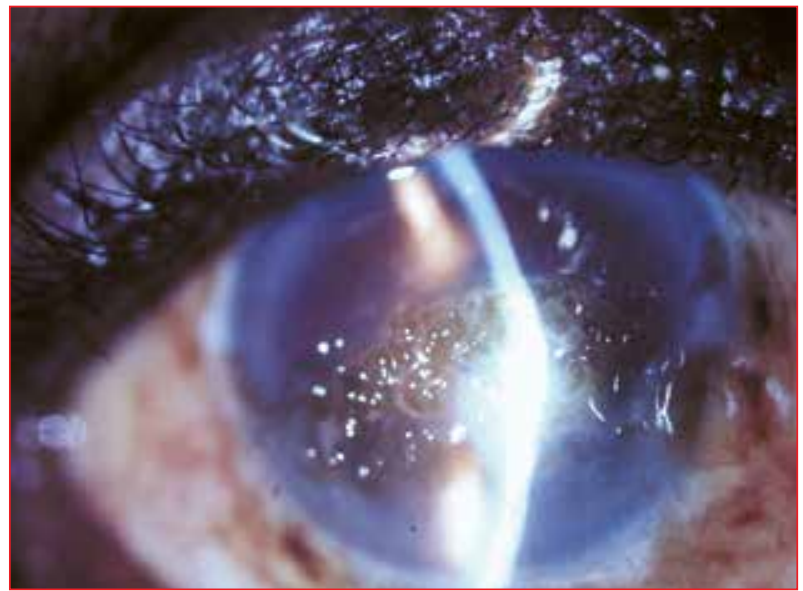

Source: The Division of Ophthalmology, University of Cape Town

Figure 7: Uneven corneal surface in climatic droplet keratopathy

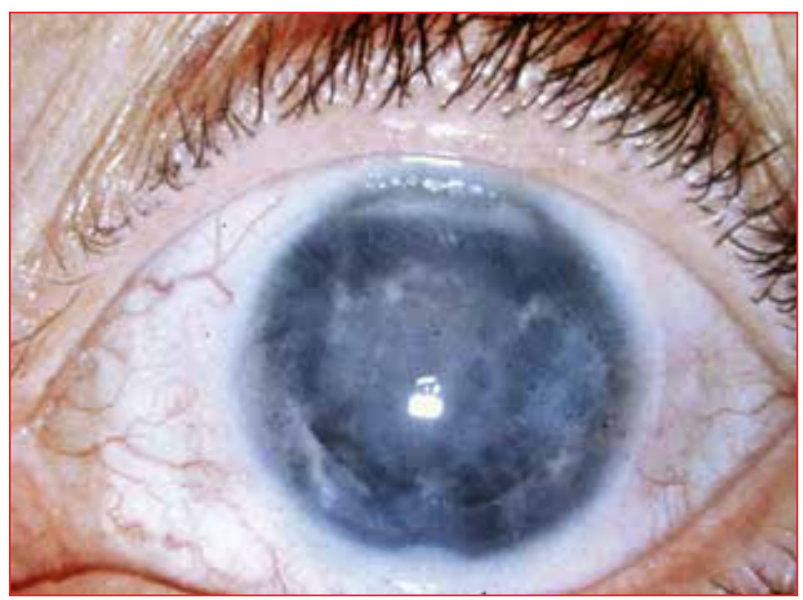

Source: The Division of Ophthalmology, University of Cape Town

Figure 8: Iris details obscured by corneal scarring

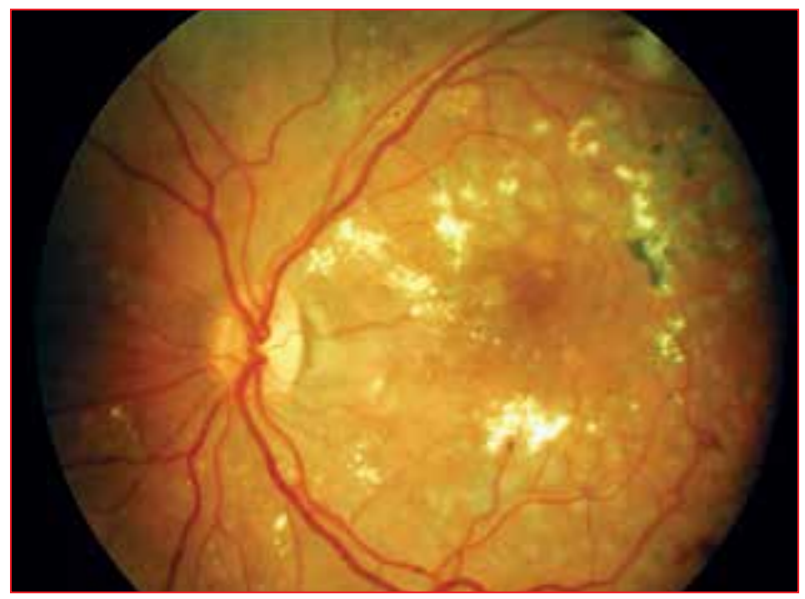

Source: The Division of Ophthalmology, University of Cape Town

Figure 9: Laser scars visible in the macular area in a patient with diabetic maculopathy

opacification is usually easily diagnosed by the presence of a reduction in the red reflex, with underlying iris details not being clear in the area of opacification (Figure 8). Generally, corneal grafting is needed to remove the opacified, scarred corneal tissue and to restore vision. 


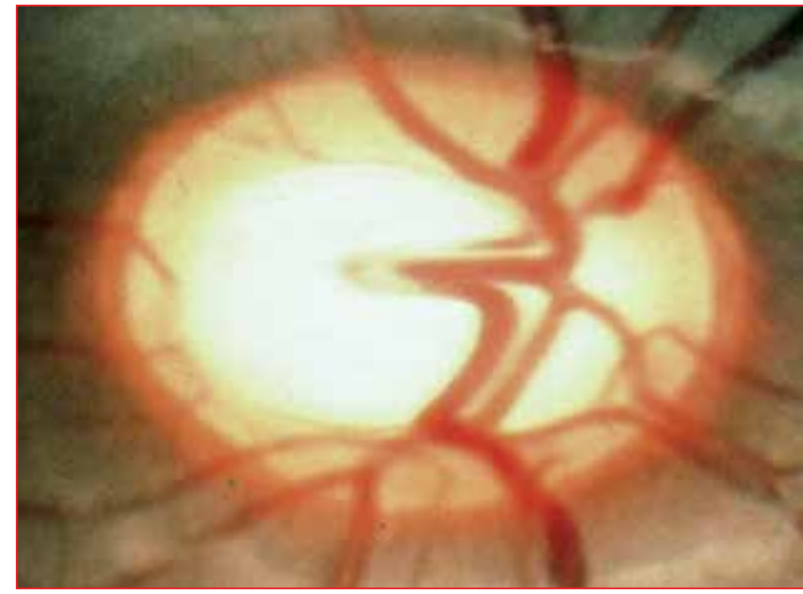

Source: The Division of Ophthalmology, University of Cape Town

Figure 10: Cupping of the optic disc in glaucoma

\section{Diabetic maculopathy}

Macular oedema may occur in diabetes, leading to insidious loss of vision, which can be prevented with appropriate laser therapy (Figure 9). This topic will be covered in another paper on systemic disease and the eye.

\section{Optic atrophy}

Pallor of the optic disc results from damage to the nerve fibres at any point from the retina to the lateral geniculate body. Patients have poor vision with an afferent pupil defect, decreased colour vision and usually a central visual field defect. Causes of optic atrophy include compression of the optic nerve, e.g. tumour; damage to the optic nerve head in chronic glaucoma; central retinal artery occlusion; and metabolic, infective and genetic diseases of the retina, e.g. retinitis pigmentosa, syphilis, tobacco-alcohol amblyopia, ethambutol toxicity and chronic papilloedema. Headaches, focal neurological signs or endocrinological signs should alert the clinician to possible intracranial mass lesions. ${ }^{1}$

Clinically, disc pallor on fundoscopy may be divided into three groups:

- Glaucomatous cupping (Figure 10).

- Diffuse pallor of the disc in non-glaucomatous optic atrophy.

- A raised, pale disc (Figure 11) in optic atrophy, following disc swelling. ${ }^{3}$

\section{Glaucoma}

Glaucoma is defined as an "optic neuropathy" with cupping of the optic disc, a characteristic pattern of visual field loss, and in most cases, raised intraocular pressure (IOP). ${ }^{3}$ Measured by tonometry, normal IOP is $10-21 \mathrm{mmHg}$. Visual acuity and IOP may be normal in a patient with glaucoma. ${ }^{8}$ Glaucoma is classified as acute or chronic, depending on the rate of onset, and primary or secondary, depending on whether or not there is an underlying ocular cause. This can further be divided into open or closed angle, depending on

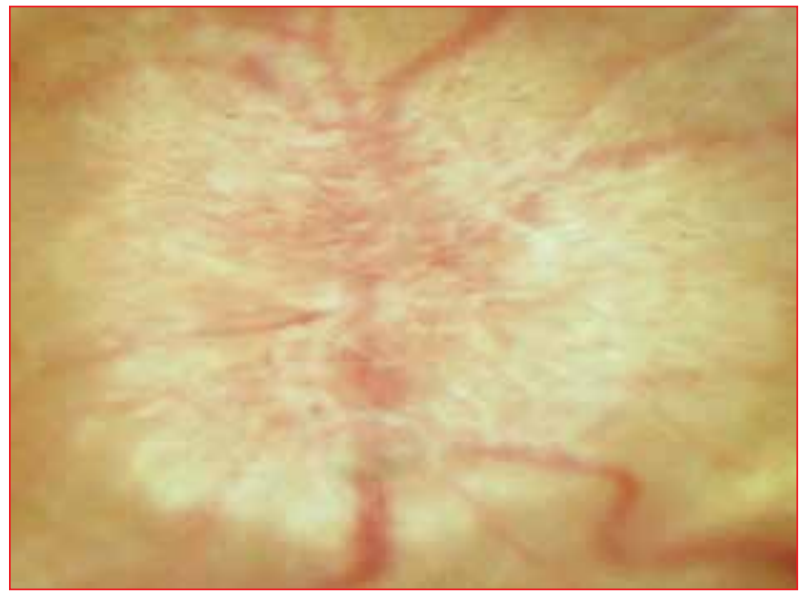

Source: The Division of Ophthalmology, University of Cape Town

Figure 11: Blurred disc margins with pallor, indicating atrophy secondary to swelling

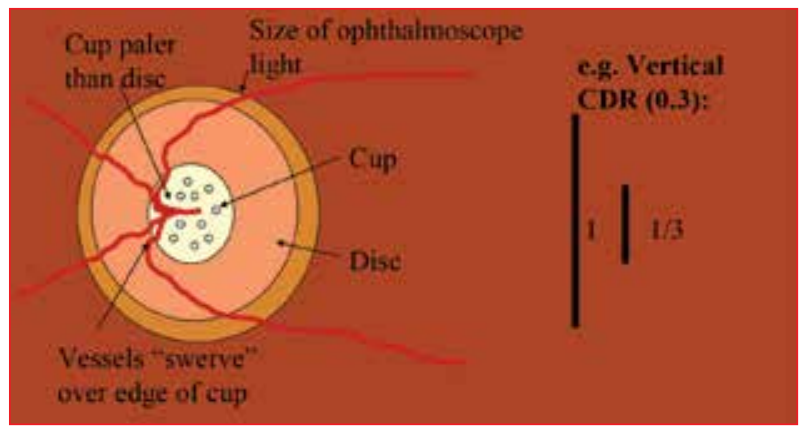

CDR: cup-to-disc ratio

Figure 12: Diagrammatic presentation of cup-to-disc ratio as assessed on fundoscopy

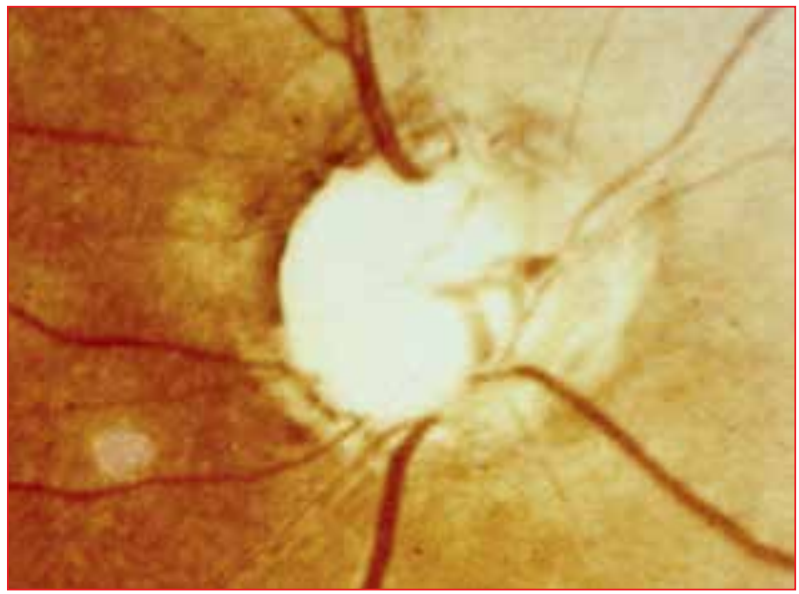

Source: The Division of Ophthalmology, University of Cape Town

Figure 13: Disc pallor due to end-stage cupping

the gonioscopic findings during assessment of the anterior chamber angle. The most common type is chronic primary open-angle glaucoma. It is an asymptomatic disease with insidious onset, and is a result of trabecular resistance to aqueous outflow. ${ }^{8}$ Patients with raised IOP, but no optic neuropathy or field loss, are referred to as ocular hypertensives. Underlying ocular causes of secondary glaucomas may be cataracts, trauma, inflammation or neoplasias. Risk factors for primary open-angle 


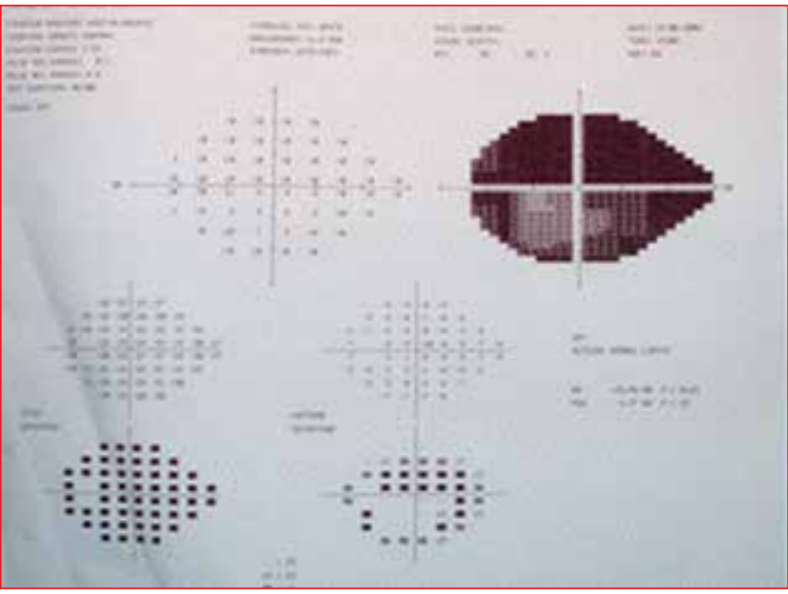

Source: The Division of Ophthalmology, University of Cape Town

Figure 14: Print out from automated visual field analyser, showing advanced loss

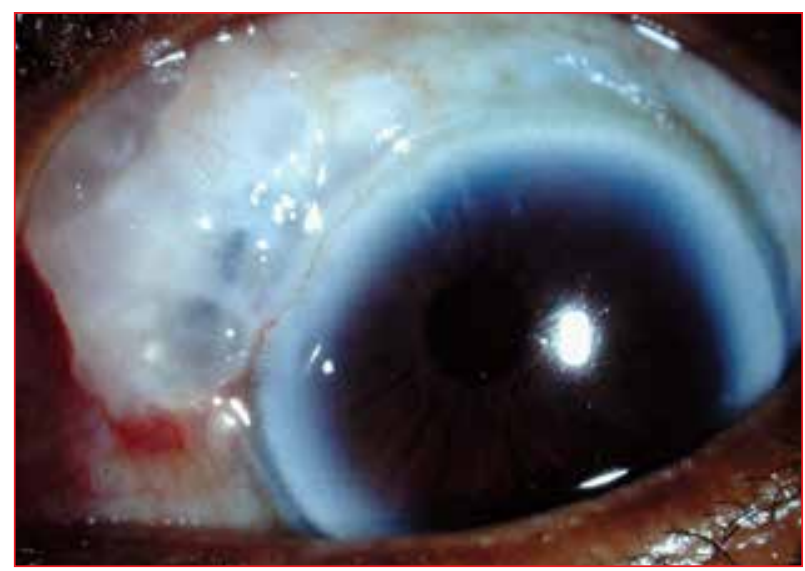

Source: The Division of Ophthalmology, University of Cape Town

Figure 15: Conjunctival bleb after trabeculectomy

glaucoma include genetic [trabecular meshwork-inducible glucocorticoid response (TIGR) myocillin gene], being of Black African ancestry, having myopia, being over 45 years of age, and having diabetes and uncontrolled hypertension (possibly). ${ }^{3}$

Symptoms vary, but generally do not include pain. Visual acuity may be normal early on (asymptomatic) as central vision is preserved until late in the disease, or the patient may be blind on presentation. Glaucoma is diagnosed by looking at the disc, as well as assessing the fields and IOP. The optic cup is a pale depression in the centre of the disc. The disc is made up of neural tissue. A cup-to-disc ratio of $>0.6$ is suggestive of glaucomatous cupping, in which the loss of nerve fibres leads to pathological enlargement of the cup (Figure 12).

The following are also signs of glaucomatous cupping: baring, bayoneting and nasalisation of the disc vessels; disc haemorrhages; exposed lamina cribrosa (laminar dot sign); notching of the cup and disc pallor (Figure 13). ${ }^{3}$

Loss of visual field in glaucoma follows the pattern of the retinal nerve fibre layer, and these defects are detected by automated perimetry using a field analyser (Figure 14).

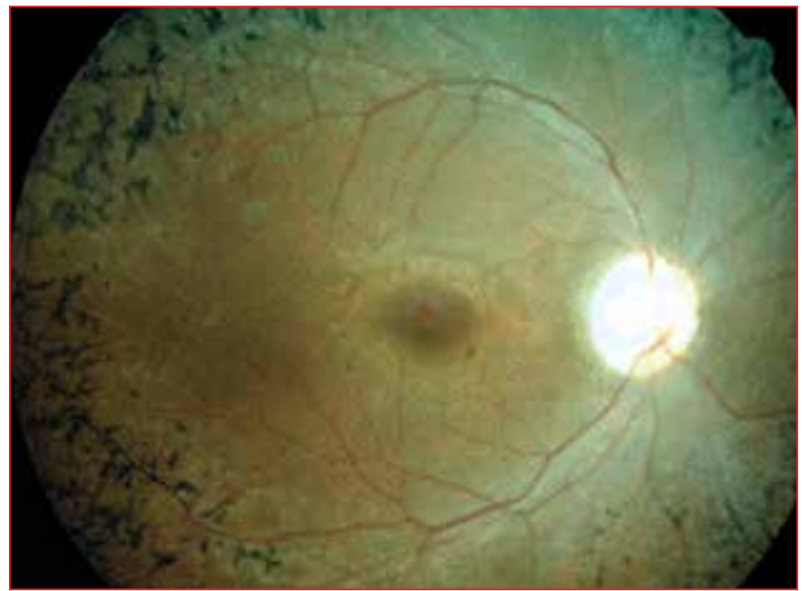

Source: The Division of Ophthalmology, University of Cape Town

Figure 16: Fundus photograph illustrating the triad of signs in retinitis pigmentosa

Tunnel vision is found in advanced cases and eventually ends in blindness.

Management includes referral to an ophthalmologist for treatment and regular follow-up. Patients are monitored for progression of cupping and field loss, which can be prevented by lowering the IOP. ${ }^{8}$ This can be achieved medically with topical agents (drops), e.g. $\beta$ blockers and prostaglandin analogues, or surgically with drainage procedures, e.g. trabeculectomy (Figure 15). Patients with a family history of glaucoma in a first-degree relative should be referred for screening. ${ }^{1}$

\section{Retinitis pigmentosa}

This is the most common inherited retinal condition, and occurs because of dystrophy of the photoreceptors. Patients complain of night blindness, tunnel vision and eventual blindness. Features include a "waxy" pallor of the disc, bone-spicule pigmentary retinopathy and attenuated retinal blood vessels (Figure 16). Retinitis pigmentosa has many systemic associations, including deafness, heart block and cerebellar disease. Many different genetic mutations are responsible. Most types are not treatable, but those associated with metabolic disorders may be arrested by treatment of the metabolic abnormality, and patients need to be referred for diagnosis, genetic counselling and social services. ${ }^{1}$

\section{Papilloedema}

This refers to disc swelling that is usually bilateral and due to raised intracranial pressure. Acute papilloedema does not cause loss of vision, but may cause visual obscurations that last a few seconds and which occur several times a day. Chronic papilloedema can cause slow loss of vision because of optic atrophy. If the disc is swollen and the patient complains of sudden loss of vision, papillitis (optic neuritis) should be suspected, and the patient referred to an ophthalmologist urgently. It is important to look for 


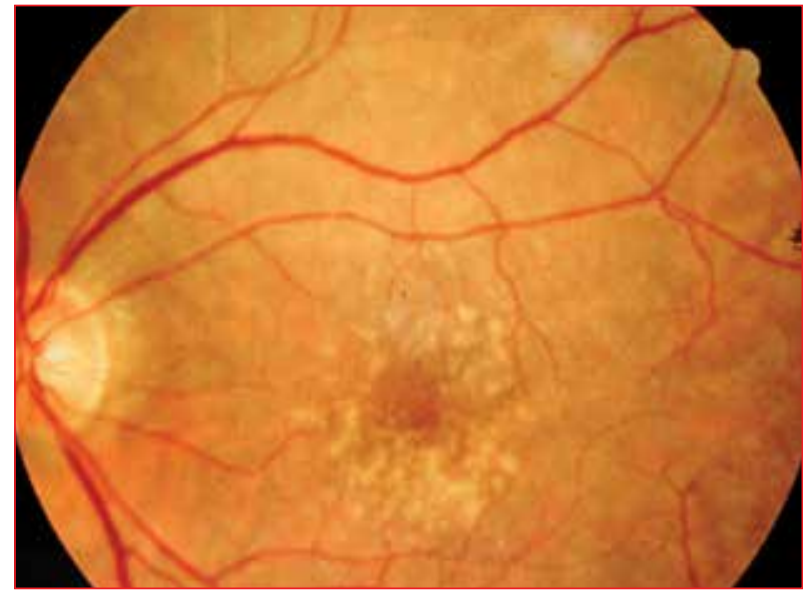

Source: The Division of Ophthalmology, University of Cape Town

Figure 17: Drusen around the fovea (centre of the macula)

papilloedema in patients with symptoms of nausea, early morning headaches and personality changes, as well as those with sixth cranial nerve palsies. ${ }^{9}$ The patient should be referred to the neurologist for imaging. Investigation is indicated in these cases.

\section{Age-related macular degeneration}

Age-related macular degeneration is a disease of the elderly and is common in Caucasians. Symptoms and signs which patients may experience include slow loss of vision ("dry" type), sudden loss of vision from bleeding ("wet" type) and metamorphopsia (distorted images). ${ }^{3}$ Age-related macular degeneration is characterised by drusen, which are pale deposits in the macular area (Figure 17), and which are very common in the elderly. Initially, drusen do not interfere with vision. The atrophy of the retinal pigment epithelium, with resultant central pigmentary changes, occurs in dry agerelated macular degeneration. Haemorrhage and exudation from subretinal neovascular membranes occurs in wet age-related macular degeneration (Figure 18). Age-related macular degeneration is a very common cause of bilateral visual loss in the developed world, ${ }^{10}$ especially in patients over the age of 65 years. Central vision is affected, but peripheral vision spared, thus allowing continued patient mobility.

Patients with age-related macular degeneration who develop loss of central vision due to leakage and bleeding from subretinal neovascular membranes, may benefit from laser therapy or intravitreal injection of antivascular growth factor agents. ${ }^{3}$ Visual loss is usually bilateral, but may be asymmetrical. Patients with established visual loss require "low vision aids", e.g. magnifiers, and should be referred to low vision services, as well as considered for blind registration and other support. ${ }^{10}$

\section{Chronic ocular inflammation}

Band keratopathy (opaque calcific corneal deposits) (Figure 19) may be a sign of chronic intraocular inflammation which

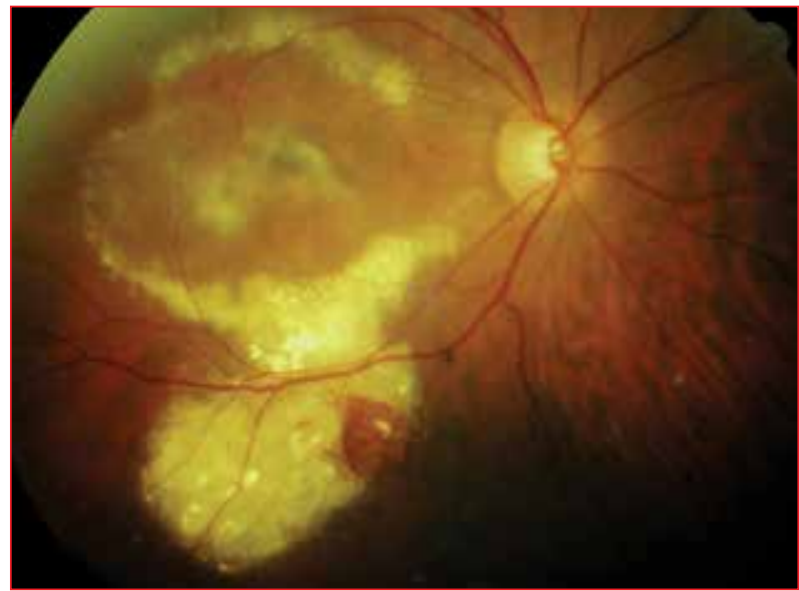

Source: The Division of Ophthalmology, University of Cape Town

Figure 18: Exudates and haemorrhage in "wet" age-related macular degeneration

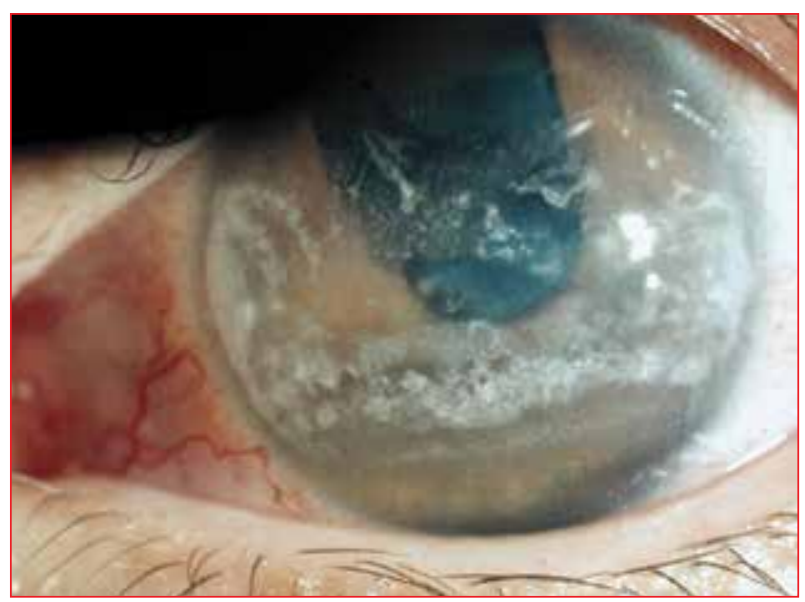

Source: The Division of Ophthalmology, University of Cape Town

Figure 19: Calcific corneal deposits in band keratopathy

results in calcification of the cornea, e.g. with chronic uveitis. Juvenile idiopathic arthritis is associated with uveitis and causes painless, slow loss of vision, without causing a red eye. These children should have regular eye examinations. Children with pauci-articular rheumatoid factor-negative, anti-nuclear antibody-positive arthritis, are at highest risk. ${ }^{3}$

\section{Conclusion}

Gradual loss of vision is a common problem at primary care level, especially in older patients. Cataracts are the most cause of loss of vision. Diagnosis is easily made in most cases. Appropriate referral and management generally result in good outcomes, except in those with irreversible visual loss from optic atrophy with its various causes, and possibly also in those with macular degeneration. Newer treatments of age-related macular degeneration have improved prognosis in many cases, and this field is expanding. There is hope for improved treatment of this condition in the future. ${ }^{11}$ 


\section{Acknowledgements}

The Division of Ophthalmology of the University of Cape Town is acknowledged for providing permission to use its clinical photographs.

\section{Conflict of interest}

There is no conflict of interest to declare.

\section{References}

1. Elkington AR, Khaw PT. Gradual visual loss, partial sight and blindness. BMJ. 1988;297(6647):548-549.

2. Van Zyl LM, Fernandes N, Rogers G, Du Toit N. Primary health eye knowledge among general practitioner's in the Cape Town metropole. S Afr Fam Pract. 2011;53(1):52-55.
3. Du Toit N, Cook C. Gradual loss of vision. Cape Town: Juta; 2009.

4. Salomao SR, Cinoto RW, Berezovsky A, et al. Prevalence and causes of vision impairment and blindness in older adults in Brazil: the Sao Paulo Eye Study. Ophthalmic Epidemiol. 2008;15(3):167-175.

5. Murthy GV, Vashist P, John N, et al. Prevalence and causes of visual impairment and blindness in older adults in an area of India with a high cataract surgical rate. Ophthalmic Epidemiol. 2010;17(4):185-195

6. Pascolini D, Mariotti SP. Global estimates of visual impairment: $2010 . \mathrm{Br} \mathrm{J}$ Ophthalmol. 2012;96(5):614-618.

7. Morgan IG, Ohno-Matsui K, Saw SM. Myopia. Lancet. 2012;379(9827):1739-1748.

8. Quigley HA. Glaucoma. Lancet. 2011;377(9774):1367-1377.

9. Selhorst JB, Chen Y. The optic nerve. Semin Neurol. 2009;29(1):29-35.

10. Siemsen DW, Brown WL. Vision rehabilitation of persons with age related macular degeneration. Semin Ophthalmol. 2011;26(3):65-68.

11. Kaufman SR. Developments in age-related macular degeneration: diagnosis and treatment. Geriatrics. 2009;64(3):16-19.

\section{Master's Degree in Clinical Pharmacology}

\section{MPharmMed}

Acquire a critical and analytical approach to clinical pharmacology, and develop your therapeutic reasoning and decision-making skills.

The MPharmMed course comprises a three-year, part-time course and covers all aspects of clinical pharmacology, namely pharmacokinetics, pharmacodynamics, toxicology and medical biostatistics. Topics such as evidence-based medicine, pharmaco-economics and the critical appraisal of literature are included. A research project must also be completed, with the aim of applying research methodology in different work environments. The course has been structured into various modules that are also individually accredited for CPD purposes. There is a strong emphasis on clinical research, which will open doors to other medical and pharmaceutical career opportunities for the degree holder.

The MPharmMed degree is presented by the Department of Pharmacology at the University of Pretoria. It is unique in South Africa and has, since 1974, provided a singular opportunity for doctors practising in all areas of medicine to follow a formal course in clinical pharmacology. The popularity of this degree has grown over the years, emphasising the importance of clinical pharmacology in modern medicine.

The next three-year course commences in January 2014.

Please contact Mrs J Bekker at (012) 319 2243, or julia.bekker@up.ac.za for further information. Alternatively, write to the Department of Pharmacology, School of Medicine, Faculty of Health Sciences, University of Pretoria, Private Bag X323, Arcadia, 0007.

Please note that full registration with the HPCSA is a requirement for enrolment. 